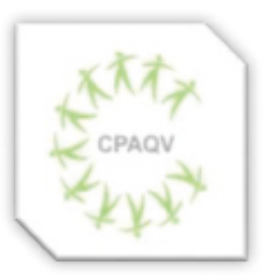

ISSN: 2178-7514

Vol. $12\left|\mathbf{N}^{\circ} .3\right|$ Ano 2020
ARTIGO ORIGINAL

\section{AGILIDADE E FLEXIBILIDADE EM PRATICANTES DE CROSSFIT}

\section{Agility and flexibility in crossfit practitioners}

Cristiano Luiz Soares ${ }^{1}$; Marina Avayou Brito ${ }^{2}$; Renan Fernandes da Cunha ${ }^{2}$; Adriano de Almeida Pereira ${ }^{3}$; Juliana Cristina Viola ${ }^{3}$; Uebister Igor dos Santos Guedes ${ }^{3}$; Mateus Valério de Almeida; Alexandre Freitas de Carvalho ${ }^{3}$; Ricardo Pablo Passos ${ }^{3}$; Guanis de Barros Vilela Junior ${ }^{3}$; Hugo Politano ${ }^{3}$

\title{
RESUMO
}

Atualmente existem diversos box de CrossFit espalhados no Brasil e no mundo. Diante disso, o presente estudo teve como objetivo identificar e comparar a agilidade e a flexibilidade entre praticantes de CrossFit da cidade de Lavras no Sul de Minas Gerais. A amostra foi composta por 18 praticantes de CrossFit, sendo 10 do sexo masculino (grupo 1) e 8 do sexo feminino (grupo 2), numa faixa etária de 20 a 29 anos, onde todos atenderam os critérios de inclusão. O estudo é descritivo de cunho transversal e delineamento comparativo. Os resultados encontrados para a flexibilidade masculino e feminino respectivamente, tiveram média e desvio padrão de $32,9 \pm 8 \mathrm{~cm}$ e $35,8 \pm 5 \mathrm{~cm}$, e na agilidade de $9,8 \pm 0,4$ e 11,6 $\pm 1,3$ segundos. Conclui-se que para na variável flexibilidade não existe diferenças significativas entre os grupos, $\mathrm{p}=0,389$, no entanto, na agilidade foi observada diferença significativa entre os grupos de $\mathrm{p}=0,001$ para praticantes regulares de CrossFit.

Palavras-chave: CrossFit. Flexibilidade. Agilidade.

\begin{abstract}
Currently there are several CrossFit boxes spread throughout Brazil and the world. Therefore, this study aimed to identify and compare the agility and flexibility among CrossFit practitioners in the city of Lavras in southern Minas Gerais. The sample was composed of 18 CrossFit practitioners, 10 male (group 1) and 8 female (group 2), in an age range of 20 to 29 years, where all met the criteria for inclusion. The study is a cross-sectional descriptive and comparative design. The results found for male and female flexibility respectively, had mean and standard deviation of $32.9 \pm 8 \mathrm{~cm}$ and $35.8 \pm 5 \mathrm{~cm}$, and in agility of $9.8 \pm 0.4$ and $11.6 \pm 1.3$ seconds. It is concluded that for the flexibility variable there are no significant differences between the groups, $\mathrm{p}=0.389$, however, in the agility significant difference was observed between the groups of $\mathrm{p}=0.001$ for regular CrossFit practitioners.
\end{abstract}

Keywords: CrossFit. Flexibility. Agility.

1 - ESEFIC - Escola Superior de Educação Física de Cruzeiro SP

2 - Centro Universitário do Sul de Minas - Grupo Unis

3 - Universidade Metodista de Piracicaba - Unimep

Autor de correspondência

Cristiano Luiz Soares

Email: cristianosoares.ig@gmail.com 


\section{INTRODUÇÃO}

Segundo o guia de treinamento de Nível 1 do CrossFit, o método é definido como treinos funcionais variados, que envolvem múltiplas articulações e são executados em alta intensidade. Sendo classificado como uma metodologia voltada para a melhoria da resistência cardiorrespiratória, força, flexibilidade, resistência muscular, potência, velocidade, coordenação, agilidade, equilíbrio e precisão. Dentre esses benefícios apresentados, a pesquisa dará ênfase em duas capacidades físicas, sendo elas: a agilidade e a flexibilidade. Segundo Hollmann \& Hettinger $^{(1)}$, a flexibilidade é definida como "uma qualidade física responsável pela execução voluntária de um movimento de amplitude angular máxima, por uma articulação ou conjunto de articulações, dentro dos limites morfológicos, sem risco de provocar lesão”. E a agilidade segundo Pitanga ${ }^{(2)}$ é definida pelo deslocamento do centro de gravidade do corpo e a realização de trocas rápidas de direção.

Assim, levando em consideração o aumento de adeptos e a crescente popularização do CrossFit no Brasil, torna-se cada vez mais necessários estudos sobre o tema para proporcionar informações sobre o efeito da modalidade para os praticantes e também oferecer o respaldo cientifico aos profissionais da área.

Portanto, o presente estudo tem como objetivo identificar a agilidade e a flexibilidade dos praticantes de CrossFit da cidade de Lavras, Minas Gerais.

\section{Flexibilidade}

É a capacidade máxima para mover uma articulação por uma variação de movimento. Amplitude articular máxima em uma ou mais articulações ${ }^{(3)}$.

Já Heyward ${ }^{(4)}$, define a flexibilidade na capacitação de uma articulação, ou serie de articulações, mover-se ao longo de uma amplitude de movimento completa sem lesão. A flexibilidade é um elemento importante da aptidão física relacionada a saúde, é essencial para manter a independência funcional na execução de atividades da vida diária, além de desempenhar um papel importante em numerosas modalidades esportivas. De acordo com American Collegeof Sports Medicine é uma das práticas recomendadas para a aquisição de uma melhor qualidade de vida. ${ }^{(5)}$

\section{Agilidade}

Segundo Bompa ${ }^{(6)}$ a agilidade é a capacidade física que o atleta consegue mudar de direção de forma ágil e eficaz, mover-se com facilidade no campo ou simular ações que enganem o adversário a sua frente, em sua maioria sendo determinada pela genética, mas podendo ser desenvolvidas. Referindo-se ser benéfica para a maioria dos atletas de esportes coletivos. A agilidade aumenta por meio de exercícios que exigem uma mudança rápida 
dos movimentos com participação de todo o corpo.

Barrow\&McGee ${ }^{(7)}$ afirmam ser resultado de uma capacidade inata, porém com o treino e a experiência pode ser melhorada. Relacionam a agilidade à coordenação dos grandes grupos musculares do corpo em uma atividade particular.

Para Costello\&Kreis ${ }^{(8)}$, a definição de agilidade seria a habilidade de mudar de direção sem que haja perda de força, velocidade, controle do corpo ou equilíbrio. Outra definição confirmada por Sale ${ }^{(9)}$, existem quatro razões para avaliar a agilidade: determinar sua importância no desempenho, desenvolver o perfil do atleta, acompanhar o progresso do treinamento e monitorar a reabilitação de lesões.

\section{Crossfit}

É um programa diversificado que foi criado especificamente para melhorar a saúde, tendo como intuito de desenvolver e aperfeiçoar a aptidão dos praticantes em todas as tarefas e sua forma física. Neles são realizados movimentos funcionais de alta intensidade, que visam aprimorar os movimentos ginásticos (argolas, paradas de mão, barras), capacidade aeróbia (corrida, bicicleta, remo) e exercícios do levantamento olímpico (clean, snatch, levantamento terra, entre outros). As sessões de treinamento são chamadas de "Workouts of the Day", ou WODs, normalmente envolve a combinação de movimento realizados em um formato de circuito, com pouco ou nenhum período de descanso. Alguns exercícios são realizados no menor tempo possível e outros são executados no maior número de rodadas que o praticante possa conseguir realizar. No entanto a estrutura de cada sessão varia entre cada box de afiliados, normalmente cada sessão dura cerca de sessenta minutos e compreende um trabalho específico de aquecimento, força e / ou técnica, um treino de força ou condicionamento programado por dez a trinta minutos, e resfriamento e / ou trabalho de mobilidade. ${ }^{(10,11,12,13)}$

\section{MATERIAIS E MÉTODOS}

O estudo caracteriza-se como quantitativo, descritivo e transversal. A amostra contou com indivíduos do sexo masculino (grupo 1) e feminino (grupo 2), que deveriam ter experiência com a prática de pelo menos seis meses e estarem ativos na modalidade CrossFit. O mesmo foi desenvolvido em um box, na cidade de Lavras, MG.

O estudo ocorreu de maneira voluntária, obedecendo todas as exigências da Resolução 196/96 do Conselho Nacional de Saúde e teve seu protocolo aprovado no Comitê de Ética em Pesquisa Centro Universitário do Sul de Minas.

Os indivíduos realizaram o teste de sentar e alcançar, conhecido popularmente como "Banco de Wells" para medição de sua amplitude de alongamento dos músculos posteriores da coxa e da região posterior do 
tronco. A aferição foi feita com eles sentados, com os pés apoiados no banco e os joelhos estendidos. Em seguida, o avaliador terá que segurar os joelhos do avaliado, onde seu tronco será flexionado a frente na tentativa de alcançar a maior quantidade de centímetros que puder conseguir ${ }^{(2)}$. O teste foi executado numa caixa de madeira medindo as seguintes dimensões: $30,5 \mathrm{~cm} \times 30,5 \mathrm{~cm} \times 30,5 \mathrm{~cm}$, sendo que na parte de cima, onde está localizada a escala, terá um prolongamento de $26,0 \mathrm{~cm}$ e o $23^{\circ} \mathrm{cm}$ da escala onde coincidirá com o ponto onde o indivíduo toca a planta dos pés ${ }^{(5)}$.

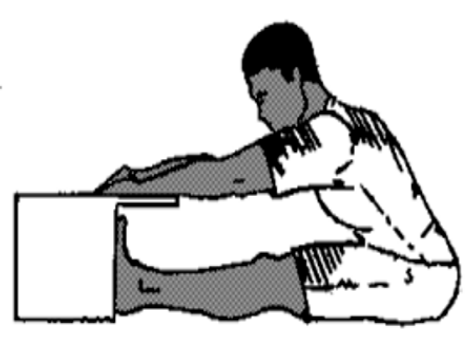

Figura 1. Teste Banco de Wells. Fonte: PROESP-BR Projeto Esporte Brasil

Já o teste Shuttle Run, foi mensurado através da corrida de 9,14 metros, onde o avaliado coloca-se na linha de partida, após ouvir o comando e o cronômetro for acionado, correrá o mais rápido possível até a outra linha para pegar o primeiro bloco $(5 \mathrm{~cm} \times 5 \mathrm{~cm} \times$ $10 \mathrm{~cm})$, voltar até a linha de partida e depositar o bloco, voltar até a outra linha pegar o segundo bloco, voltar até a linha de partida, e depositar este último bloco, ultrapassando com pelo menos com um dos pés a linha de chegada. O cronômetro foi parado no momento em que o avaliado terminou de cumprir a tarefa. Os blocos foram colocados no solo, não sendo permitido jogá-los. ${ }^{(2)}$

Realizam-se 02 (duas) tentativas, primeiro foi realizado duas tentativas do teste do Banco de Wells, seguido pelas duas tentativas do teste Shuttle Run, foram considerados o melhor valor alcançados. As realizações de ambos os testes foram aferidas nos mesmo dia e em um lugar apropriado para a coleta, não havendo nenhum tipo de aquecimento antes de realiza-los.

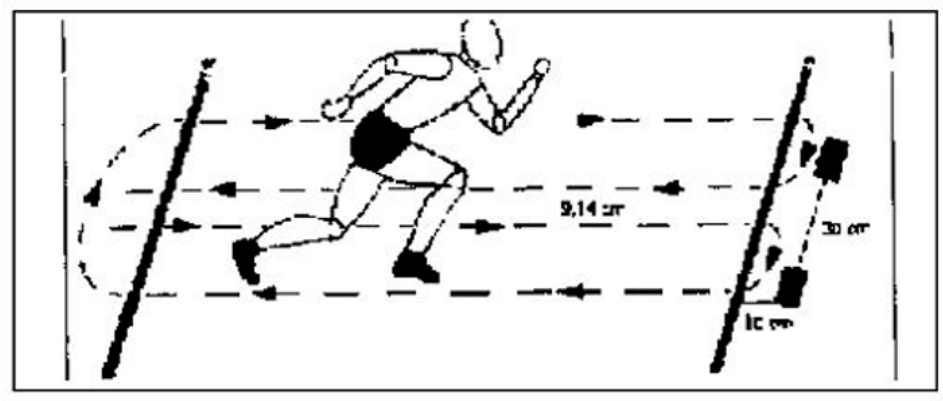

Figura 2. Teste de ShuttleRun. 


\section{RESULTADOS E DISCUSSÃO}

No teste para avaliação da flexibilidade o grupo 1 (Masc) apresentou uma média aritmética de $32,9 \mathrm{~cm}$ alcançados no banco e wells com um desvio padrão de $8 \mathrm{~cm}$, e 9,8 segundos (seg.) no teste de agilidade, com desvio padrão de 0,4 seg..

No grupo 2 (Fem), o resultado foi para a capacidade de flexibilidade $35,8 \mathrm{~cm}$ de média aritmética com desvio padrão de $5 \mathrm{~cm}$, e 11,6 seg. na agilidade, este com um desvio padrão de 1,3 seg.

Tabela 1. Média aritmética e desvio padrão referentes das capacidades flexibilidade e agilidade dos praticantes de Crossfit.

\begin{tabular}{lccc}
\hline Grupos & Idade & Flexibilidade & Agilidade \\
\hline 1 (Masc) & $25 \pm 2,8$ & $32,9 \pm 8$ & $9,8 \pm 0,4$ \\
2 (Fem) & $23,6 \pm 2,3$ & $35,8 \pm 5$ & $11,6 \pm 1,3$ \\
\hline
\end{tabular}

Tabela 2. Análise comparativa da variáveis Flexibilidade, Agilidade e Idade nos diferentes grupos avaliados praticantes de Crossfit.

\begin{tabular}{lcc}
\hline & $F$ & Sig \\
\hline Flexibilidade & 0,784 & 0,389 \\
Agilidade & 18,554 & 0,001 \\
ldade & 1,246 & 0,281 \\
\hline
\end{tabular}

Fonte: Software SPSS 20.0

$\mathrm{Na}$ análise comparativa entre as mulheres é normalmente imposta devido as médias aritméticas dos grupos nas diferentes diferenças sexuais na estrutura pélvica e aos variáveis avaliadas foi considerado um grau hormônios que podem afetar a lassidão do de confiança de 95\%. Todavia, admiti-se que tecido conectivo. A flexibilidade está referente apenas para a variável Agilidade foi identificada diferença significativa entre os grupos, com ao tipo corporal, ao sexo idade, a idade e ao nível de atividade física. p-0,001, contudo, não foram identificadas

Segundo Konkol e Kronbauer ${ }^{(15)}$ a diferenças significativas na idade, $p=0,281$, e na flexibilidade, $\mathrm{p}=389$.

Para Achour ${ }^{(14)}$, o sexo feminino é mais flexível do que o sexo masculino em todas as idades, justifica-se talvez pelas atividades que exigem maior uso de flexibilidade do sexo feminino e pelas atividades de força predominantes pelo sexo masculino (4), evidência que a maior flexibilidade das flexibilidade sofre interferência por questões anatômicas e hipertrofia muscular entre os sexos. Os homens em geral são beneficiados em executar serviços que envolvem força e velocidade. No entanto, com a prática regular e sistematizada do treinamento é possível, conforme resultados apresentados, estabelecer bons resultados na variável, uma vez que a mesma pode ser modulada conforme o tipo de 
trabalho realizado.

Já em um outro estudo envolvendo atletas de futsal a nível escolar com praticantes de outras modalidades que se encaixem nas mesmas categorias e descrições. A amostra teve a participação de 172 alunos de ambos os sexos, e foram divididos em três grupos: 15 anos, 16 anos e 17 anos. Em conclusão, o estudo definiu que em relação a comparação do futsal com as outras modalidades ambos os sexos encontram-se no mesmo grau de classificação na questão de flexibilidade (Banco de Wells), foram considerados como excelentes e acima da média, e a uma deficiência demostrada pelos avaliados no quesito agilidade (Shuttle Run), ambos os sexos foram classificados como fracos. ${ }^{(16)}$

De acordo com trabalho de Drews et al. ${ }^{(17)}$ foram analisados a agilidade de 60 indivíduos do sexo masculino, com idade média de $16 \pm 0.4$ anos, na qual um grupo praticava futsal e o outro praticava voleibol. Os resultados mostraram que não apresentaram nenhuma diferença estatisticamente significativa entre os grupos analisados.

Castro et al. (18) foram aplicados os testes Shuttle Run e Banco de Wells antes e após a intervenção em mulheres praticantes de hidroginástica com a faixa etária de 44 a 59 anos, durante 8 semanas. Os resultados demonstraram que houve melhoras significativas para as variáveis motoras: flexibilidade e agilidade.

No entanto, Silva et al. ${ }^{(19)}$ analisaram participantes de ambos os sexos que possuíam idade entre 11 e 14 anos, e foram divididos em grupo teste e grupo controle não praticantes de Taekwondo. As sessões tiveram uma durabilidade de 12 semanas, as avaliações foram realizadas na fase pré e pós prática. Um dos testes utilizados foram Teste de Sentar e Alcançar no Banco de Wells, e o Shuttle Run. Os Resultados mostraram que os praticantes do Taekwondo, dos sexos feminino e do masculino melhoraram os níveis das variáveis de flexibilidade e agilidade, porém o mesmo não ocorreu no grupo controle.

Por conseguinte, no estudo retratado por Carlos ${ }^{(20)}$, foi realizado o teste de flexibilidade (Banco de Wells) para comparar os parâmetros biomecânicos relacionados aos músculos do core. As coletas foram realizadas em 32 participantes do sexo feminino com idade entre 18 e 30 anos, e que possuíssem ao menos 6 meses de treinamento nas modalidades CrossFit, pilates ou musculação e com indivíduos sedentários. Os valores da flexibilidade para os três treinamentos não apresentaram diferença significativa. Quando se observa o grupo de sedentarismo este sim apresentou uma diferença significativa, o que seria um resultado já esperado, ficando abaixo das outras modalidades.

Eather et al. ${ }^{(21)}$ com 96 participantes $(15,4(0,5)$ anos, foram realizados os testes de Shuttle Run e de sentar e alcançar no início e pós-intervenção, durante 8 semanas, tendo como objetivo de avaliar a eficácia preliminar e viabilidade do treinamento de resistência em adolescentes. Os resultados através dos testes mostraram sucesso na melhoria da aptidão relacionada à saúde, tantos nas meninas e nos meninos, sendo sugerido que incorporem o 
treinamento CrossFit no currículo das aulas de educação física, visando ser uma boa abordagem para promover a aptidão física em adolescentes.

\section{CONCLUSÃO}

Conclui-se que para ambos o sexo (masculino e feminino), praticante da modalidade de Crossfit da cidade de Lavras, não houve diferença significativa quanto a flexibilidade provavelmente pela interferência do meio, mostrando a treinabilidade da variável, no entanto, verificou-se diferença significativa quanto a agilidade.

Sugere-se novos estudos com praticantes da mesma modalidade de cunho transversal e longitudinal, para que se possa identificar possíveis melhoras em condições de treinamento.

\section{REFERÊNCIAS}

1. Hollmann, W., \& Hettinger, T. (2005). Medicina do Esporte: Fundamentos anatômico-fisiológicos para a prática esportiva. Manole.

2. Pitanga, F.J.G. (2008). Teste, medidas e avaliação em educação física e esportes. 5 ed. São Paulo: Phorte.

3. Fontoura, A. S., Formentin, C. M., \& Abech, E. A. (2011). Guia prático de avaliação física: uma abordagem didática, abrangente e atualizada. Phorte Editora LTDA.

4. Heyward, H. (2004). Avaliação Física e Prescrição de Exercício Técnicas Avançadas trad. Márcia Dornelles.

5. Novaes, G. Da Silva; Mansur, H.; Nunes, R. A. M. (2011). Grupos especiais: avaliação, prescrição e emergências clínicas em atividades físicas. 1 ed. São Paulo: Icone.

6. Bompa, T. O. (2002). Treinamento Total para Jovens Campeões. Tradução de Cássia Maria Nasser. Revisão Científica de Aylton J. Figueira Jr.

7. Barrow, H.M.; McGee, R. (1978). A practical approach to measurement in physical education. Philadelphia: Lea \&Febiger.

8. Costello, F.; Kreis, E.J.D. (1993). Sports agility. Tenesse: Taylor Sports.

9. Sale, D.G. Testing strength hand power. In: MacDougall, V.D.; Wenger, H.A.; Green, H.J. Physiological testing of the high performance athlete. Champaing: Human Kinetics, 1991. p.21-106.
10. Butcher S. J; Neyedly T. J; Horvey K. L; BENKO C. R. Do physiological measures predict selected CrossFit ${ }^{\circledR}$ benchmark performance? Open Access Journal of Sports Medicine. 2015:6.

11. Smith, M.M; Sommer, A.J; Starkoff, B.E; Devor, S.T. (2013). Crossfit based high-intensity power training improves maximal aerobic fitness and body composition. Journal of Strengt hand Conditioning Research.

12. Crossfit. O Guia de Treinamento de Nível 1 do CrossFit. 2007. <<Disponível em: http://library. crossfit.com/free/pdf/CFJ_L1_TG_Portuguese.pdf $>>$ Acesso em 02 de maio de 2018.

13. Tibana R.A; DE Almeida L.M; Prestes J. (2015). Crossfit ${ }^{\circledR}$ riscos ou benefícios? $O$ que sabemos até o momento. Revista BrasileiraCiênciae Movimento. Vol. 23. Num. 1. p. 182-185.

14. Achour Junior, A. (1996). Bases para exercícios de alongamento relacionado com a saúde e no desempenho atlético. Londrina: Midiograf.

15. Konkol, A. Kronbauer, G.A. Componentes da aptidão física dos dançarinos de um grupo folclórico polonês. Cinergis 2011;12(1):1-7.

16. Nascimento, G.S. (2013). Análise das Capacidades Físicas em Atletas de Futsal. Trabalho de Conclusão de Curso (Graduação em Educação Física) Universidade Católica de Brasília, Brasília.

17. Drews, R.; Cardozo, P.L.; Corazza, S.T.; Flores, F.S. (2013). Análise do desempenho motor de escolares praticantes de futsal e voleibol. FTCD/FIP-MOC.

18. Castro, C. L.; Assumpção, C.O; Bartholomeu Neto, J.; Asano, R.Y. (2008). Efeitos da prática da Hidroginástica nas capacidades Físicas de Mulheres de 44 a 50 anos de idade. Anuário de Produção Acadêmica Docente Anhanguera Educacional. Vol. XII.

19. Silva, J.F; Silvestre, T. F.; Silva, G.R.; Terra, G.D.S.V.; Tavares M. R.; Neiva, C.M.N.; Boas, Y.F.V.; Rodrigues, C.A.C. (2017). Análise comparativa da flexibilidade, agilidade e força de membros inferiores em adolescentes praticantes e não praticantes de Taekwondo.ConexãoCi. Vol. 3.Research Gate.

20. Carlos, L.C. Analise Biomecânica dos Músculos do Core em Praticantes de Diferentes Modalidades de Treinamento. 2016. 66f. Dissertação (Mestrado pelo programa de Pós-Graduação em Desenvolvimento Humano e Tecnologias) - Instituto de Biociências, Universidade Estadual Paulista, Rio Claro.

21. Eather, N.; Morgan, P. J.; Lubans, D. R. (2016). Improving health-related fitness in adolescents: the CrossFit Teens ${ }^{\mathrm{TM}}$ randomized controlled trial. Journal of sports sciences, v. 34, n. 3, p. 209-223.

OBSERVAÇÃO: Os autores declaram não existir conflitos de interesse de qualquer natureza. 